\title{
Estudo longitudinal da densidade mineral óssea em cães jovens da raça Golden Retriever: Correlações com idade e peso corpóreo
}

Giglio Fortes ROBSON ${ }^{1}$ Júlio César de Carvalho BALIEIRO $^{2}$

Franklin de Almeida

STERMAN ${ }^{1}$

Ana Carolina Brandão de Campos Fonseca PINTO ${ }^{1}$

Maria Angélica MIGLINO ${ }^{1}$

Mayana ZATZ3

Cássio Ricardo Ferrigno

AUADA $^{1}$

Correspondência para: ROBSONFORTES GIGLIO

Av. Marte, 125, apto 33 - Alphaville 06483-310 - Santana de Parnaíba-SP rgiglio@usp.br

Recebido para publicação: 13/12/2004 Aprovado para publicação: 01/05/2006

\author{
1 - Departamento de Cirurgia da Faculdade de Medicina Veterinária e \\ Zootecnia da Universidade de São Paulo, São Paulo - SP \\ 2 - Faculdade de Zootecnia e Engenharia de Alimentos da Universidade de \\ São Paulo, Pirassununga - SP \\ 3 - Instituto de Biociências da Universidade de São Paulo, São Paulo - SP
}

\section{Resumo}

A Densitometria Óptica Radiográfica é um método de análise para quantificação da matéria mineral óssea, sendo, neste estudo, realizado com radiografias simples da tíbia direita ao lado de escala de alumínio. Foram utilizados 5 cães da raça Golden Retriever, que foram radiografados mensalmente, dos 3 aos 9 meses de idade. Estas radiografias foram digitalizadas e analisadas por meio de um software de análise de imagens (ImageLab, Softium ${ }^{\circledR}$ ) que compara as tonalidades de cinza da escala com região óssea analisada, obtendo-se valores em milímetros de alumínio ( $\mathrm{mmAl}$ ). $\mathrm{O}$ estudo revelou que a região epifisária possui maior densidade mineral óssea (DMO), seguida pela região metafisária e diafisária, respectivamente, ao longo do período experimental, e seguiram o comportamento do peso corpóreo. Houve uma tendência de aumento da DMO nas três regiões avaliadas. A região metafisária proximal de tíbia demonstrou ser a região de eleição para a leitura da DMO em cães em crescimento por ser o local estudado com menor correlação com o peso corpóreo, possibilitando uma maior influência de outros fatores na regulação da DMO, como alguma alteração que promova osteopenia, gerando um diagnóstico mais precoce.

\section{Introdução}

O tecido ósseo promove suporte para as partes moles, protege órgãos vitais, aloja a medula óssea, atua como um depósito de cálcio, fosfato e outros íons, proporciona apoio aos músculos esqueléticos e é constituinte do sistema de alavancas que ampliam as forças geradas na contração muscular ${ }^{1}$.

Sofre contínua remodelação, desenvolvendo-se em proporção direta ao estresse sofrido, tornando-se mais espesso quando submetido à carga de peso maior ${ }^{2}$.

O nível de atividade e exercícios são, principalmente para pessoas saudáveis e jovens, fatores predominantes na regulação da densidade mineral óssea ${ }^{3}$. Slemenda et al. ${ }^{4}$ também sugeriram que importante aumento da densidade óssea pode ser resultado da realização de atividades físicas durante a infância.

O exame radiográfico simples não é eficiente na detecção de alterações do processo de mineralização óssea inferior a $30 \%$ de seu conteúdo 5 .

A densidade mineral óssea pode ser estimada através de métodos invasivos e não-invasivos, que são divididos em métodos radiológicos e não-radiológicos ${ }^{6}$.

A densitometria óptica radiográfica enquadra-se nos métodos não-invasivos radiológicos porém possui um custo significativamente inferior aos demais ${ }^{6,7}$, sendo sugerida por Louzada et al. ${ }^{8}$ para transpor as limitações visuais e a subjetividade 
foram realizados. Muramoto 9 estudou a raça Poodle e Vulcano et al. ${ }^{10} \mathrm{em}$ um estudo piloto e Alves ${ }^{11}$ obtiveram os valores normais de densidade mineral óssea da extremidade distal do rádio da raça Rottweiller.

Originalmente utilizado para a busca de presas abatidas em ambientes aquáticos e alagadiços, a escolha da raça Golden Retriever para este estudo deve-se ao grande crescimento e popularidade desta raça no território brasileiro, ficando em décimo lugar em número de registros, com 2550 exemplares registrados no ano de $2003^{12}$, e, diferente dos estudos citados no parágrafo anterior, o objetivo deste estudo em questão não foi determinar valores normais da densidade mineral óssea para esta raça, e sim comparar os valores encontrados nas diferentes regiões estudadas com o peso corpóreo e a idade, de forma longitudinal.

\section{Materiais e Métodos}

Foram utilizados 5 cães da raça Golden Retriever nascidos no canil do Setor de Anatomia dos Animais Domésticos e Silvestres do Departamento de Cirurgia da Faculdade de Medicina Veterinária e Zootecnia da Universidade de São Paulo.

Estes animais foram submetidos a um programa de adoção, o qual previa retornos mensais, quando eram pesados e radiografados, a partir dos três meses de idade até o fechamento do disco epifisário proximal da tíbia direita com 9 meses de idade.

O estudo da densidade mineral óssea foi realizado por meio de radiografia simples da tíbia direita paralela e simultaneamente a escala de alumínio confeccionada com liga específica e padronizada internacionalmente (Liga de alumínio 2026, ABNT).

Os animais foram radiografados em decúbito lateral direito, em incidência médiolateral, utilizando-se o aparelho de raio X da marca Ray-tec modelo RT 500/125, com chassis de $18 \times 24 \mathrm{~cm}$, com écrans intensificadores Kodak Lanex X-Omatic Regular Screens (Kodak Eastman
Company) e filme radiológico no tamanho $18 \times 24 \mathrm{~cm}$, RP-X-OMAT (Kodak Bras. Com. E Ind. Ltda).

A técnica radiográfica utilizada nos dois primeiros meses de estudo foi de: quilovoltagem de 40 quilovolts $(\mathrm{kV})$, miliamperagem de 100 miliampères $(\mathrm{mA})$, tempo de exposição de 0,025 segundos (s) e distância foco-filme de $100 \mathrm{~cm}$ para todos os animais. Em virtude do crescimento dos animais, a partir do terceiro mês de estudo, correspondente ao quinto mês de idade, até o seu término a técnica radiográfica foi ajustada para: quilovoltagem de 44 quilovolts $(\mathrm{kV})$, miliamperagem de 100 miliampères (mA), tempo de exposição de 0,025 segundos (s) e distância foco-filme de 100 $\mathrm{cm}$.

A revelação dos filmes foi feita em processadora automática, RP-X-OMAT Processor (Kodak Eastman Company).

$O$ processo de digitalização da imagem radiográfica foi feito através do scanner de mesa Hewlett-Packard, modelo Scanjet 6300C, com adaptador para transparências Hewlett-Packard, modelo Scanjet XPA.

As imagens foram salvas em escala de cinzas no modo bmp (bitmap) pelo software HP PrecisionScan Pro e armazenadas no disco rígido do microcomputador Pentium III de $450 \mathrm{MHz}$, HD de $11 \mathrm{~Gb}$ de memória, $128 \mathrm{Mb}$ de memória RAM, Infoway (Itautec ${ }^{\circledR}$ ).

As imagens radiográficas, digitalizadas e convertidas em uma escala em tons de cinzas no programa de informática HP PrecisionScan Pro $\left(\right.$ Hewlett-Packard $\left.^{\mathbb{}}\right)$, após isto foram processadas e analisadas com o auxílio do programa ImageLab (Softium ${ }^{\circledR}$, Sistemas de Informática), que compara a densidade média da região do osso selecionada com a densidade média dos degraus da escala de alumínio, determinando a equivalência em milímetros de alumínio da densidade da região selecionada (Figuras 1 e 2).

Neste estudo, três regiões da tíbia foram estudadas: epífise proximal da tíbia, metáfise proximal da tíbia e diáfise proximal 
da tíbia.

Com o intuito de que a densidade mineral óssea fosse a mais fidedigna possível, deve-se padronizar que a área selecionada.

Para demarcar a região epifisária proximal o quadrilátero utilizado foi o trapézio, com sua base maior posicionada proximal ao disco epifisário, contemplando toda a região epifisária.

Para demarcar a região metafisária proximal o quadrilátero utilizado foi o losango, com um de seus lados, de mesmas medidas, posicionado distal ao disco epifisário.

Para demarcar a região diafisária proximal o quadrilátero utilizado foi o retângulo, iniciando-se 3 centímetros distal a região metafisária, com altura correspondente a medida entre os bordos da região estudada e largura correspondente ao dobro da altura.

Em todas estas demarcações incluise uma pequena extensão de partes moles com o intuito de se subtrair a densidade das partes moles sobrepostas.

Com as regiões de interesse demarcadas, foi calculado, por meio do programa de informática, o valor de densidade óptica em milímetros de alumínio de cada região. Para se obter um valor mais fidedigno, a demarcação e cálculo da densidade óptica de cada região foram realizados três vezes, obtendo-se uma média dos valores obtidos nas leituras (Figura 3).

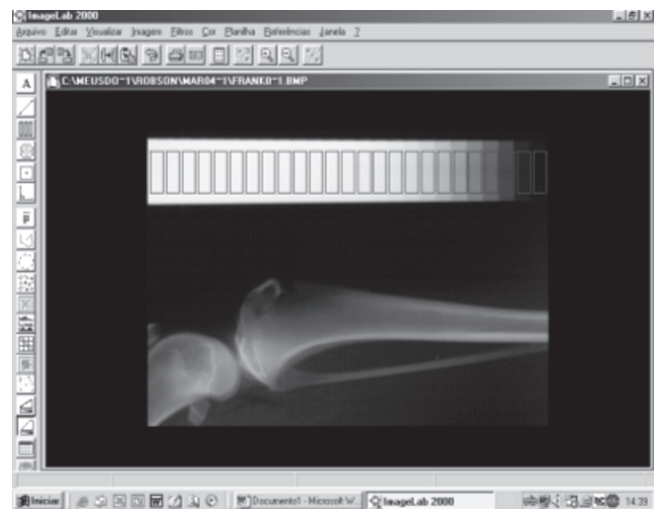

Figura 1 - Visualização do programa ImageLab mostrando a seleção dos 25 degraus da imagem padrão do penetrômetro (Estudo longitudinal da densidade mineral óssea em cães jovens da raça Golden Retriever: Correlações com idade e peso corpóreo)

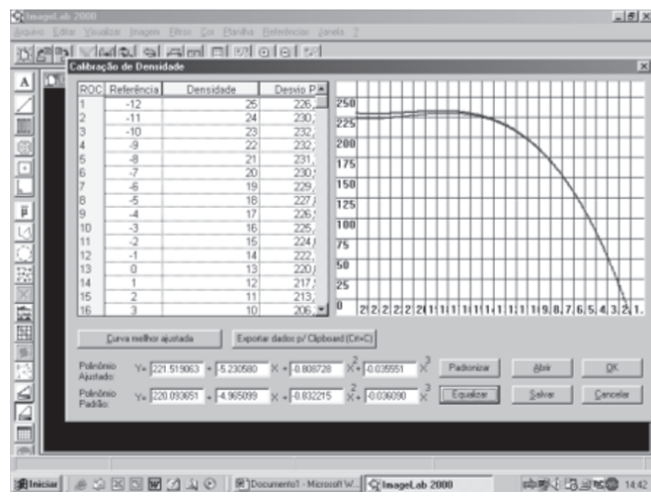

Figura 2 - Visualização do programa ImageLab mostrando a planilha e o gráfico após a seleção dos degraus do penetrômetro de outra radiografia, onde se observa no gráfico uma curva em vermelho representando os valores de densidade óptica radiográfica dos degraus da imagem padrão e outra curva em azul representando os valores de densidade óptica radiográfica dos degraus de outra radiografia, anterior ao processo de equalização desta nova imagem a imagem estabelecida como padrão (Estudo longitudinal da densidade mineral óssea em cães jovens da raça Golden Retriever: Correlações com idadee peso corpóreo)

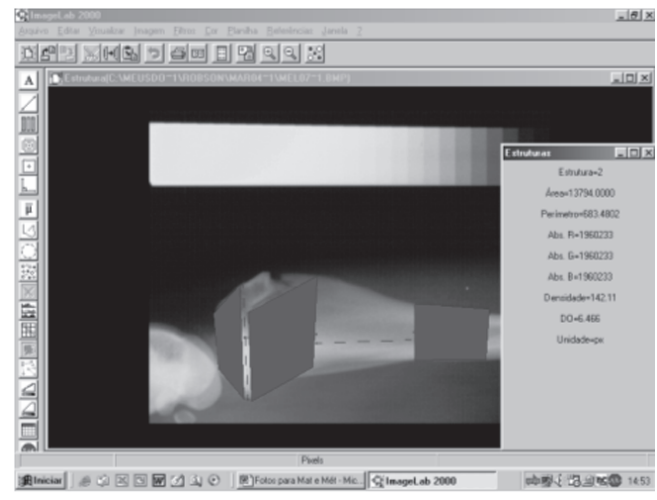

Figura 3 - Visualização do programa ImageLab mostrando a seleção dos três quadriláteros correspondentes as regiões epifisária $(\mathrm{E})$ metafisária (M) e diafisária(D) com a leitura da densidade óptica (DO), à direita, da região metafisária (M) (Estudo longitudinal da densidade mineral óssea em cães jovens da raça Golden Retriever: Correlações com idade e peso corpóreo)

Para obtenção da estatística descritiva para as variáveis peso corpóreo $(\mathrm{em} \mathrm{Kg})$ e os valores da densidade mineral óssea das três regiões analisadas (em mmAl) utilizou-se o procedimento PROC MEANS do programa Statistical Analysis System, versão $8.0^{13}$.

Para a análise de correlação entre as regiões ósseas analisadas com o peso corpóreo nas diferentes idades (em meses) no período experimental utilizou-se o Teste 
Tabela 1 - Estimativas médias do peso corpóreo (Kg) e densidades minerais ósseas (em mmAl) das regiões epifisária, metafisária e diafisária proximais da tíbia direita dos cães da raça Golden Retriever dos 3 aos 9 meses de idade, São Paulo, 2004

\begin{tabular}{ccccc}
\hline Idade $(\mathrm{m})$ & Peso $(\mathrm{Kg})$ & $\begin{array}{c}\text { DMO epifisária } \\
(\mathrm{mmAl})\end{array}$ & $\begin{array}{c}\text { DMO diafisária } \\
(\mathrm{mmAl})\end{array}$ \\
\hline 3 & 2,200 & 2,439 & 1,775 & 1,585 \\
4 & 3,540 & 3,463 & 2,928 & 2,535 \\
5 & 8,875 & 6,689 & 5,453 & 4,478 \\
6 & 14,875 & 6,835 & 5,435 & 4,356 \\
7 & 20,950 & 7,256 & 5,774 & 4,935 \\
8 & 22,650 & 7,912 & 6,289 & 5,079 \\
9 & 24,950 & 8,591 & 6,558 & 5,770 \\
\hline
\end{tabular}

Tabela 2 - Análise de correlação, segundo teste de Pearson, entre os valores médios da densidade mineral óssea nas diferentes regiões analisadas com a idade (em meses) dos cães Golden Retriever ao longo do período experimental, São Paulo, 2004

\begin{tabular}{cl}
\hline & Idade $(\mathrm{m})$ \\
\hline DMO epifisária $(\mathrm{mmAl})$ & 0,93227 \\
DMO metafisária $(\mathrm{mmAl})$ & 0,91112 \\
DMO metafisária $(\mathrm{mmAl})$ & 0,93348
\end{tabular}

Tabela 3 - Análise de correlação, segundo teste de Pearson, entre os valores médios da densidade mineral óssea nas diferentes regiões analisadas com o peso corpóreo (em Kg) dos cães Golden Retriever, São Paulo, 2004

\begin{tabular}{cl}
\hline & Peso $(\mathrm{Kg})$ \\
\hline DMO epifisária $(\mathrm{mmAl})$ & 0,92617 \\
DMO metafisária $(\mathrm{mmAl})$ & 0,90427 \\
DMO metafisária $(\mathrm{mmAl})$ & 0,92135 \\
\hline
\end{tabular}

de Pearson do procedimento PROC CORR do mesmo programa supracitado.

\section{Resultados}

Os resultados obtidos neste estudo foram expressos nas tabelas 1,2 e 3 .

\section{Discussão}

O processamento das imagens, a possibilidade de equalização dos tons de cinzas do penetrômetro da radiografia a ser analisada com a radiografia do penetrômetro escolhido como padrão, a delimitação precisa da região escolhida, e a verificação de valores muito próximos nas três medidas de cada região permitem concluir que a técnica da Densitometria Óptica Radiográfica mostrou ser de fácil execução, rápida, de baixo custo, precisa, sensível, confiável e reprodutível, como afirmam outros autores que utilizaram o mesmo programa computacional ImageLab $b^{9,11,14,15} \mathrm{e}$ outros que utilizaram a mesma técnica densitométrica com outros programas de processamento de imagens $6,7,16,17,18,19,20$, viabilizando sua realização na Medicina Veterinária.

O maior incremento na densidade mineral óssea foi observado entre o quarto e o quinto mês, que foi acompanhado por maior incremento do peso corpóreo, como evidenciado pela alta correlação (superiores a 0,9$)$ demonstrada pela análise estatística em todas as regiões analisadas. 
As regiões epifisária e diafisária proximais da tíbia obtiveram valores de correlação altos e próximos, tanto em relação ao peso corpóreo quanto a idade.

Embora seja alta (superior a 0,9), a região metafisária proximal da tíbia foi a região que obteve menor correlação com o peso corpóreo e a idade entre as estudadas. Este fato pode ser explicado pelo fato de que esta região, nos animais em crescimento, possui tecido ósseo imaturo, o qual tem uma menor mineralização ${ }^{1}$ e grande atividade metabólica neste período etário avaliado.

Embora composta quase que totalmente por osso compacto, delimitando o canal medular, a diáfise possui menor densidade mineral óssea em relação às outras porções ósseas. Isto ocorre porque quando se seleciona esta região, o programa calcula a média dos tons de cinzas na área selecionada para elaboração do valor densitométrico final incluindo o canal medular que possui menor radiopacidade.

Por meio destes dados, observou-se que o peso corpóreo possui grande relação com os valores da densidade mineral óssea nas diferentes regiões ósseas analisadas, em concordância com outros autores ${ }^{9,11,16,19}$.

Os valores médios de densidade mineral óssea na região epifisária foram mais elevados, seguidos pela região metafisária e diafisária e, respectivamente, houve um incremento mensal da densidade mineral óssea das três regiões estudadas.

\section{Conclusões}

A região epifisária possui maior densidade mineral óssea, seguida pela região metafisária e diafisária, respectivamente, ao longo do período experimental.

Os valores da densidade mineral óssea (DMO) das três regiões avaliadas, observando-se pequenas diferenças de correlação, seguiram o comportamento do peso corpóreo ao longo do período experimental.

A região metafisária proximal de tíbia demonstrou ser a região de eleição para a leitura da densidade mineral óssea (DMO) por ser o local estudado com menor correlação com o peso corpóreo nos cães em crescimento, podendo evidenciar, de forma mais precoce, alterações que promovam osteopenia, necessitando de rigorosa padronização de seus valores normais, como já salientado por outros autores.

\section{Longitudinal study of bone mineral densitometry in young Golden Retriever dogs: Correlations with age and body weight}

\section{Abstract}

Radiographic Optical Densitometry is an analitic method to meansure bone mineral content. This technique was performed by right tíbia simple radiograph conjugated with an aluminium stepwedge in 5 Golden Retriever dogs. Monthly, all the dogs were radiographed from 3-mouths until 9-mouths old. Radiographs were digitalized and analyzed by an image processing software (ImageLab, Softium), which compares the aluminium grey scale tonalities with a bone region analyzed obtaining with this the value of bone mineral density in aluminium milimeters ( $\mathrm{mmAl}$ ). The epifisary region possess higher bone mineral density (BMD) followed for the metafisary and diafisary regions, respectively, during the experimental period and followed by the behavior of the corporeal weight. There was a trend of increase of the bone mineral density. The proximal metafisary region of tíbia, in growth dogs, demonstrated to be the best region of selection to evaluate the bone mineral density since it is place studied with less correlation with the corporeal weight, and for be enable to anothers

\section{Key-words:} Densitometry. Radiology.

Dogs.

Golden Retriever. 
factors influences in the regulacion of BMD, like some disease who promote osteopenia, producing a earlier diagnosis.

\section{Referências}

1 JUNQUEIRA, L. C.; CARNEIRO, J. Tecido ósseo. In: JUNQUEIRA, L. C.; CARNEIRO, J. Histologia básica. Rio de Janeiro: Guanabara-Koogan, 1990. p. 101-119.

2 GUYTON, A. C.; HALL, J. E. Tratado de fisiologia médica. Rio de Janeiro: Guanabara-Koogan, 1997. p. 895-910.

3 WHALEN, R. T.; CARTER, D. R. Influence of physical activity on regulation of bone density. Journal of Biomechanics, v. 21, n. 10, p. 825-837, 1988.

4 SLEMENDA, C. W. et al. Role of physical activity in development of skeletal mass in children. Journal of Bone Mineral Research, v. 6, n. 11, p. 1227-1233, 1991.

5 GARTON, M. J. et al. Can radiologist detect osteopenia on plain radiographs ?. Clinical Radiology, v. 49, p. 118-122, 1994.

6 LOUZADA, M. J. Q. et al. Avaliações de densidade óssea em imagens radiográficas: estudo em peças ósseas de cães. RBE - Caderno de Engenharia Biomédica, v. 14, n. 1, p. 47-64, 1998.

7 LOUZADA, M. J. Q. et al. Densidade de peças ósseas de frangos. Estudo pela densitometria óptica radiográfica. Veterinária e Zootecnia, v. 9, p. 95-109, 1997.

8 LOUZADA, M. J. Q. et al. Metodologia para a avaliação de densidade em imagem radiográfica. RBE Caderno de Engenharia Biomédica, v. 14, n. 2, p. 3747, 1998.

9 MURAMOTO, C. Estabelecimento de valores de densidade mineral óssea (DMO) das regiões diafisárias do rádio em cães da raça Poodle por meio da densitometria óptica radiográfica. 2003. 125 f. Dissertação (Mestrado em Cirurgia) - Faculdade de Medicina Veterinária e Zootecnia, Universidade de São Paulo, São Paulo, 2003.

10 VULCANO, L. C. et al. Determination of normal values of density of the radius in Rottweilers, using radiographic optical densitometry (an experimental study). In: CONGRESS OF THE WORLD SMALL ANIMAL VETERINARY ASSOCIATION, 23., 1998, Buenos Aires. Anais... Buenos Aires: WSAVA, 1998. p. 767.

11 ALVES, J. D. S. Estudo da densidade mineral óssea na extremidade distal do rádio de cães da raça Rottweiler, por meio da densitometria óptica radiográfica. 2004. 70 f. Dissertação (Mestrado em Anatomia dos Animais Domésticos e Silvestres) Faculdade de Medicina Veterinária e Zootecnia, Universidade de São Paulo, São Paulo, 2004.

12 CASTRO, S. M. L. Registro genealógico por raças.
Confederação Brasileira de Cinofilia (CBKC), 2004. Disponível em: <http://www.cbkc.org/ relatorio6.html >. Acesso em: 02 dez. 2004.

13 SAS. User's guide: basic and statistic. Cary: SAS, 1995. $1.686 \mathrm{p}$.

14 PRADO FILHO, J. R. C. Avaliação da densidade mineral óssea em potros da raça Puro Sangue Inglês em início de treinamento. 2001. 43 f. Dissertação (Mestrado em Cirurgia) - Faculdade de Medicina Veterinária e Zootecnia, Universidade de São Paulo, São Paulo, 2001.

15 STERMAN, F. A. Avaliação da densidade mineral óssea em eqüinos atletas destinados ao enduro eqüestre. 47 f. 2002. Tese (Livre Docência) - Faculdade de Medicina Veterinária e Zootecnia, Universidade de São Paulo, São Paulo, 2002.

16 LEAL, A. C. R. Determinação dos valores normais da densidade mineral óssea (DMO) da extremidade distal do rádio em cães por meio da técnica de densitometria óptica radiográfica em imagens radiográficas: correlação entre o peso, sexo e idade. 2002. 43 f. Dissertação (Mestrado em Cirurgia) Faculdade de Medicina Veterinária e Zootecnia, Universidade Estadual Paulista, Botucatu, 2002.

17 VULCANO, L. C. et al. Valores normais da densidade óssea do carpo ulnar em potros em crescimento da raça Quarto de Milha através da densitometria óptica radiográfica. A Hora Veterinária, v. 17, n. 100, p. 5254, 1997.

18 RAHAL, S. C. et al. Densitometria óptica radiográfica na avaliação do hiperparateoidismo secundário nutricional induzido em gatos jovens. Ciência Rural, v. 32, n. 3, p. 421-425, 2002.

19 SANTOS, F. A. M. Determinação dos valores normais da densidade mineral óssea (DMO) da extremidade distal do rádio-ulna em gatos, por meio da técnica de densitometria óptica radiográfica em imagens radiográficas: correlação entre o peso, sexo e idade. 2002. 49 f. Dissertação (Mestrado em Cirurgia) Faculdade de Medicina Veterinária e Zootecnia, Universidade Estadual Paulista, Botucatu, 2002.

20 VULCANO, L. C. et al. Determinación de los valores normales de la densidad mineral ósea del carpo accesorio de eqüinos de la raza Puro Sangre Inglês (P.S.I.) por médio de la densitometria óptica em imagen radiográfica. Imagen Veterinaria, v. 3, n. 6, p. $237-$ 240, 2000.

21 HAYASHI, T. et al. Evaluation of bone density in newborn infants by computed X-ray densitometry. Journal of Pediatric Gastroenterology and Nutrition, v. 23, p. 130-134, 1996.

22 YANG, S. et al. Radiographic absorptiometry for 
bone mineral measurements of the phalanges: precision and accuracy study. Radiology, v. 192, p. 857-859, 1994.

23 OWEN, M. Meansurements of the variations in calcification in normal rabbit bone. Journal of Bone Joint Surgery, v. 388, n. 3, p. 762-769, 1956.

24 MACK, P. B.; VOSE, G. P.; NELSON, J. D. New development in equipment for the roentgenographic meansurement of bone density. American Journal of Roentnology, v. 82, n. 2, p. 303-310, 1959. 